\title{
Mechanical stimuli induce cleavage and nuclear translocation of the polycystin-1 $\mathrm{C}$ terminus
}

\author{
Veronique Chauvet, ${ }^{1}$ Xin Tian, ${ }^{2}$ Herve Husson, ${ }^{3}$ David H. Grimm, ${ }^{1}$ Tong Wang, ${ }^{1}$ \\ Thomas Hieseberger, ${ }^{4}$ Peter Igarashi, ${ }^{4}$ Anton M. Bennett, ${ }^{5}$ \\ Oxana Ibraghimov-Beskrovnaya, ${ }^{3}$ Stefan Somlo, ${ }^{2}$ and Michael J. Caplan'
}

\begin{abstract}
${ }^{1}$ Department of Cellular and Molecular Physiology, ${ }^{2}$ Department of Medicine, Section of Nephrology, Yale University School of Medicine, New Haven, Connecticut, USA. ${ }^{3}$ Genzyme Corp., Framingham, Massachusetts, USA. ${ }^{4}$ Department of Internal Medicine, University of Texas Southwestern Medical Center, Dallas, Texas, USA. ${ }^{5}$ Department of Pharmacology, Yale University School of Medicine, New Haven, Connecticut, USA.
\end{abstract}

\begin{abstract}
Polycystin-1, which is encoded by a gene that is mutated in autosomal dominant polycystic kidney disease (ADPKD), is involved in cell-matrix interactions as well as in ciliary signaling. The precise mechanisms by which it functions, however, remain unclear. Here we find that polycystin-1 undergoes a proteolytic cleavage that releases its C-terminal tail (CTT), which enters the nucleus and initiates signaling processes. The cleavage occurs in vivo in association with alterations in mechanical stimuli. Polycystin-2, the product of the second gene mutated in ADPKD, modulates the signaling properties of the polycystin-1 CTT. These data reveal a novel pathway by which polycystin-1 transmits messages directly to the nucleus.
\end{abstract}

\section{Introduction}

Autosomal dominant polycystic kidney disease (ADPKD) is one of the most common autosomal genetic disorders, affecting approximately 1 in 1,000 individuals (1). It is characterized by progressive renal cyst development, typically leading to end-stage renal disease in late middle age (1). ADPKD is caused by mutations in the PKD1 or PKD2 genes, which encode the polycystin- 1 and polycystin- 2 proteins, respectively $(2,3)$. Polycystin- 1 is a plasma membrane protein that may be involved in signaling from sites of cellcell contact or from cilia, while polycystin-2 is a transmembrane protein that can function as a cation channel (3-5). Genetic and biochemical evidence suggests that these two proteins participate in the same signaling pathway. Physical interaction between the polycystins has been demonstrated, and a mutation in either of the two genes leads to the same phenotype $(4,6,7)$. Nevertheless, the functions of these proteins and their common transduction pathway have yet to be fully elucidated.

After activation, many surface or intracellular receptors transduce signals through a series of second-messenger events ultimately leading to modulation of gene expression. However, a new signaling paradigm known as regulated intramembrane proteolysis (RIP) has been described recently (8). In this model, a cytoplasmic portion of the transmembrane receptor is released after activation and enters the nucleus, where it acts directly as a modulator of gene expression, bypassing adaptor proteins and kinase/phosphatase cascades. This phenomenon has been described for many transmembrane proteins, including cell surface receptors (Notch, APP, E-cadherin, ErbB-4, and CD44) and intracellular proteins such as SREBP,

Nonstandard abbreviations used: ADPKD, autosomal dominant polycystic kidney disease; BAC, bacterial artificial chromosome; CTT, C-terminal tail; NLS, nuclear localization sequence; $\mathrm{PKD}$, polycystic kidney disease; PLAP, placental alkaline phosphatase; PLP, periodate-lysine-paraformaldehyde; RIP, regulated intramembrane proteolysis; VSV-G, vesicular stomatitis virus major coat glycoprotein.

Conflict of interest: The authors have declared that no conflict of interest exists.

Citation for this article: J. Clin. Invest. 114:1433-1443 (2004).

doi:10.1172/JCI200421753
ATF6, and Ire1 $(8,9)$. This mechanism is conserved from bacteria to humans and controls processes as diverse as cell differentiation, lipid metabolism, and the response to unfolded proteins (reviewed in refs. 8, 9). Many proteins that undergo RIP are substrates as well for additional proteolytic cleavages that occur prior to and are required for the release of the active fragment that migrates to the nucleus. The cleavage that releases the active fragment generally occurs within the transmembrane domain of the receptor and is mediated by diverse proteases that share the characteristic of being functional in an hydrophobic environment (9).

Although polycystin- 1 is a cell surface receptor, polycystin- 2 may be located predominantly in intracellular compartments such as the ER (10) as well as at the cilium (11-13). The complex pattern of these proteins' subcellular localizations raises the possibility that they might not interact directly at the plasma membrane. Instead, their interaction may involve the relocalization of a fragment of polycystin-1 to another subcellular compartment. A cleavage that removes the $\mathrm{N}$-terminal fragment of polycystin- 1 has been reported recently (14). Here we show that polycystin-1 undergoes a cleavage that releases its C-terminal tail (CTT), suggesting that this protein might participate in a series of successive cleavages resembling the multiple proteolytic events described for the RIP mechanism. The CTT fragment of polycystin- 1 enters the nucleus and directly affects cell signaling events. Our data suggest that this signaling function is modulated by polycystin- 2 and might be initiated by mechanical stimuli.

\section{Results}

Nuclear localization of the CTT of polycystin-1 is detected in vivo in kidneys of WT embryos and transgenic mice. Three different antibodies, raised against the CTTs of human (BD3 and 46-2) and mouse (Mex-46) polycystin-1 were used to analyze the subcellular localization of this protein in mouse kidneys. The BD3 and 46-2 antibodies have been characterized previously (15). The generation of the Mex-46 antibody is described in the Methods section, and biochemical demonstration of its specificity is presented later in the text. The staining 

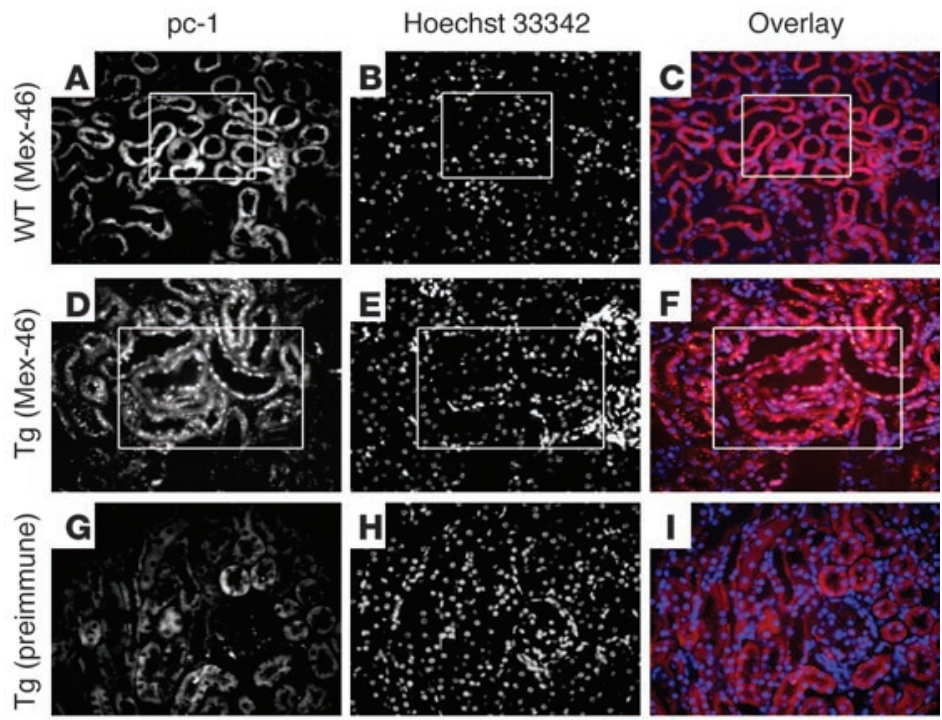

High magnification
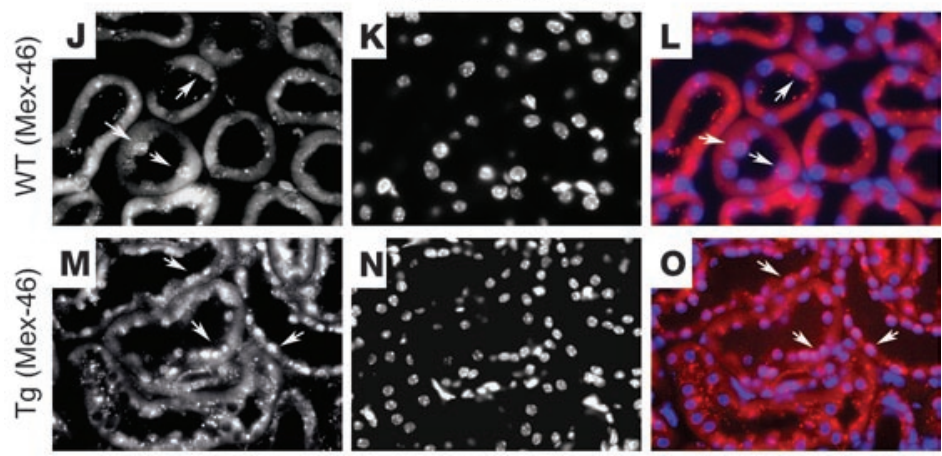

\section{Figure 1}

The CTT of polycystin-1 is detected in the nuclei of renal tubular epithelial cells in transgenic mice. (A-I) Kidneys from mice overexpressing murine polycystin-1 (Tg) (D-I) and kidneys from WT littermates $(\mathbf{A}-\mathbf{C})$ were processed for immunofluorescence using an antibody (Mex-46) specific for the CTT of polycystin-1 (pc-1) (A-F) or with the corresponding preimmune serum (G-I). Nuclei are visualized by staining with Hoechst 33342 (B, E, and H), and superposition of antibody (red) and nuclear (blue) staining is depicted in panels $\mathbf{C}, \mathbf{F}$ and I (Overlay). Nuclear staining of the polycystin-1 CTT is detected with the specific antibody (D) and not with preimmune serum (G) in the renal tubules of the transgenic mice. Little or no nuclear staining is observed in kidneys from the WT animals (A). (J-O) Higher magnification of the regions outlined in the boxes in panels $\mathbf{A}-\mathbf{F}$. Arrows indicate nuclei. Original magnifications, $\times 200(\mathbf{A}-\mathbf{I})$ and $\times 750(\mathbf{J}-\mathbf{O})$. of kidney sections presented here were obtained using Mex-46 and affinity-purified Mex-46 (Mex-46-AP). It should be noted, however, that all of the antibodies produced the same staining pattern. Immunofluorescence studies performed on the kidneys of WT mice showed a pattern of staining consistent with surface and cytoplasmic localization, as described previously $(15,16)$. However, faint nuclear staining was also detected in rare tubular segments of WT mice (Figure 1, A-C and J-L, high magnification).

To determine whether this nuclear staining was also present at early stages of nephrogenesis, we performed immunofluorescence studies of mouse embryos (13 days after conception) using Mex-46 and Mex-46-AP antibodies. At this stage of development, the level of polycystin-1 expression is reported to be higher than in it is adult specimens (17-19). A weak but widespread nuclear staining pattern was clearly observed with Mex-46 (not shown) and Mex46-AP (Figure 2, A-C and G-I, high magnification) in the condensing metanephric mesenchyme and in the developing tubules (Figure 2, A-C and G-I, high magnification), where polycystin-1 has been reported to be expressed at this stage of development $(18,19)$. No staining was detected with preimmune serum (not shown) or in the kidneys of Pkd1 KO embryos (Figure 2, D-F and $\mathrm{J}-\mathrm{L}$, high magnification).

In transgenic mice overexpressing full-length polycystin-1, in addition to the pattern described in WT adult kidneys we detected distinct nuclear staining with each of the antibodies. This pattern was observed mainly in distal segments of the nephrons
(Figure 1, D-F and $\mathrm{M}-\mathrm{O}$, high magnification). No staining was observed with preimmune sera (Figure 1, G-I) or with serum depleted of specific immunoreactivity by incubation with immobilized antigen (not shown). This pattern was observed in sections prepared from four different transgenic animals, using each of the polycystin-1 antibodies mentioned above.

These results are consistent with in vivo nuclear localization of at least a portion of the polycystin-1 intra-cytoplasmic tail. The relatively low level of nuclear detection of polycystin- 1 in kidneys of WT mice has several possible explanations. The CTT fragment might be too short-lived to be detected in the nuclei of WT renal epithelial cells, especially as the level of expression of the fulllength protein is known to be low in the tissues of adult humans and mice $(17,20)$. Preliminary results concerning the half-life of the full-length polycystin-1 obtained from the transfected MDCK cells that stably express polycystin-1 used in our study here are consistent with this hypothesis. It has been demonstrated that polycystin-2 is expressed at higher levels in adult tissues, which might regulate the distribution of the CTT, as described below. Finally, it should be noted that the nuclear localization of the Notch intracellular domain is detectable in vivo only when constitutively activated forms are expressed $(21,22)$. Thus, whereas polycystin- 1 tail is not clearly observed in the nucleus under normal circumstances, overexpression of polycystin-1 in transgenic mice results in the nuclear accumulation of quantities sufficient to produce a signal detectable by immunofluorescence. 

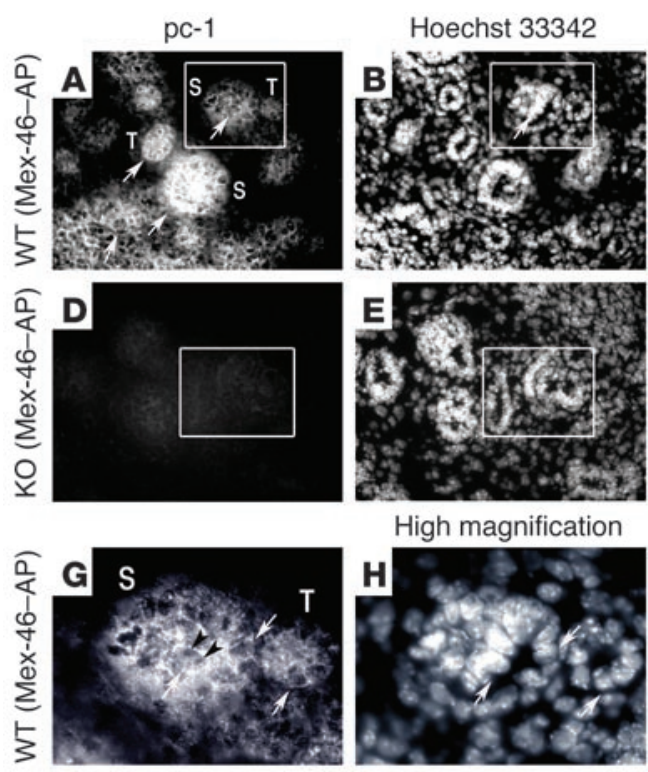

High magnification
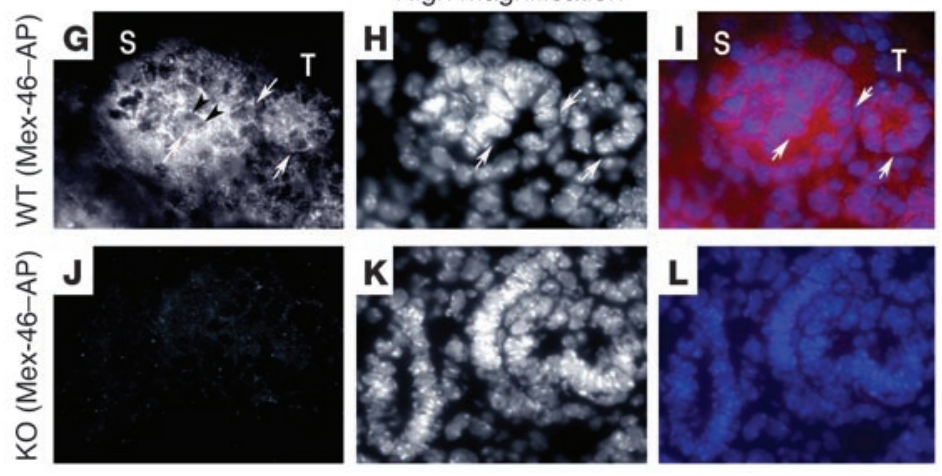
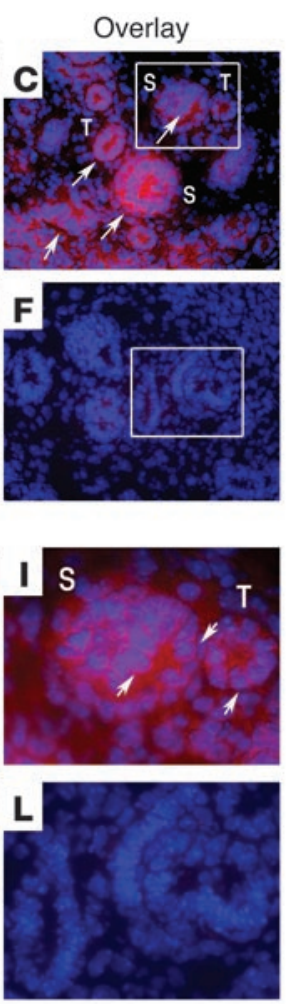

\section{Figure 2}

The CTT of polycystin-1 is detected in the nuclei of epithelial cells of developing kidneys in WT mouse embryos at 13 days after conception. (A-L) Abundant polycystin-1 staining is detected with Mex-46-AP in the condensing metanephric mesenchyme ( $S, S$ body) and developing tubules ( $T$ ) of WT mouse embryos (A-C and $\mathbf{G}-\mathbf{I})$ but not in the developing kidneys of polycystin-1 KO mice (D-F and $\mathbf{J}-\mathbf{L}$ ). The regions outlined in the boxes in panels $\mathbf{A}-\mathbf{F}$ (original magnification, $\times 200$ ) are shown at higher magnification in panels $\mathbf{G}-\mathbf{L}$ (original magnification, $\times 750$ ). Nuclear staining (arrows) is present in developing tubules of WT embryos (A and $\mathbf{G}$ ). The Mex-46-AP signal appears to be excluded from nucleoli (dark arrowheads in $\mathbf{G}$ ). Nuclei are visualized through staining with Hoechst 33342 (B, E, H, and $\mathbf{K}$ ), and superposition of antibody (red) and nuclear (blue) staining is depicted in panels $\mathbf{C}, \mathbf{F}, \mathbf{I}$, and $\mathbf{L}$ (Overlay).
The cleavage of the CTT occurs with full-length polycystin-1 in transfected cells. We tested whether the release of the CTT of polycystin-1 and its accumulation in nuclei could be detected in cells transfected with the full-length protein. The mouse full-length cDNA used for these studies was tagged at its $\mathrm{N}$ and $\mathrm{C}$ termini with Flag and HA epitopes, respectively (Figure 3A) (23). Stable MDCK cell lines expressing this construct were generated. Total cellular lysates and nuclear fractions were analyzed by Western blot. Using antibodies against either HA or Flag (not shown) or the Mex-46-AP antibody against the polycystin- $1 \mathrm{C}$ terminus, a high-molecular-weight band corresponding to the expected size of the full-length protein was detected in the total lysates (Figure 3B, arrows). In addition, a band of about $34 \mathrm{kDa}$ was distinctly present in the nuclear fractions prepared from three different MDCK clones expressing fulllength polycystin-1 with both antibody against HA (Figure 3C) and Mex-46-AP (Figure 3C). Similar results were obtained from Cos-7 and HEK cells transiently transfected with this construct (not shown). A faint band corresponding to the polypeptide of about $34 \mathrm{kDa}$ that was detected in the nuclear fractions was also visible in the blots of the total cell lysates (Figure 3B, arrowheads). The purity of the nuclear and non-nuclear fractions was demonstrated by Western blotting with antibodies against the nuclear protein histone deacetylase- 1 and the ER protein calnexin, respectively (Figure 3D). Although a very faint nuclear staining pattern was seen in MDCK cells by immunofluorescence using antibody against HA, polycystin-1 was detected predominantly in association with cytoplasmic structures and in a punctate pattern at the lateral cell surface (Figure 3E, arrow) when immunofluorescence microscopy was performed using antibody against Flag (Figure 3E). Nuclear translocation of the CTT was clearly observed in a subpopulation of Cos-7 cells transiently transfected with the full-length protein (Figure 3, F and H). This nuclear signal was not seen either by immunofluorescence (Figure 3, G and I) or by Western blotting of nuclear fractions (not shown) when antibody against Flag that recognizes the tag located at the N-terminal extremity of the protein was used. Together, these data demonstrate that release of the CTT of polycystin-1 occurs in several different cell lines stably or transiently transfected with full-length polycystin-1.

In vitro nuclear localization of a soluble cytosolic CTT of polycystin-1 and identification of a sequence necessary for its nuclear localization. To further characterize the mechanisms that lead to the nuclear localization of the CTT of polycystin-1 observed in vivo, both in the kidneys of transgenic mice and in cells expressing the full-length protein, we generated several constructs comprising portions of the human polycystin-1 cytoplasmic domain and analyzed their subcellular localization after transient transfection in several cell lines. A diagram of these constructs is shown in Figure 4A, along with their biochemical analysis by Western blotting in Figure 4B. The cytoplasmic tail of human polycystin-1 is thought to encompass approximately the last 226 residues at the $\mathrm{C}$ terminus of the protein (24). To generate a cytosolic fragment of polycystin- 1 that is not covalently linked to a transmembrane segment, we overexpressed a soluble protein containing the last 200 amino acids of human polycystin-1, which is designated the p200 construct (Figure 4A). This construct carried the Xpress epitope tag (34 additional amino acids) at its $\mathrm{N}$ terminus. The p200 polypeptide migrated by SDS-PAGE as a protein with a molecular weight of about $28 \mathrm{kDa}$, whereas the CTT derived from full-length polycystin-1 migrated as a protein with a molecular weight of about $34 \mathrm{kDa}$ (Figure 4B). When expressed in Cos-7 cells, p200 produced a strong nuclear staining pattern in immunofluorescence experiments using antibodies specific either for the $\mathrm{C}$ terminus of polycystin-1 or for the Xpress tag (Figure 4C, left panel). This staining colocalized with the staining pattern produced by an antibody directed against the nuclear marker histone 
A
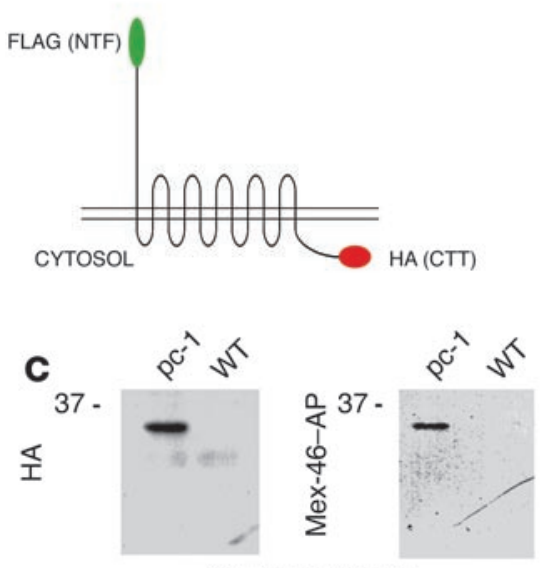

Nuclear extracts
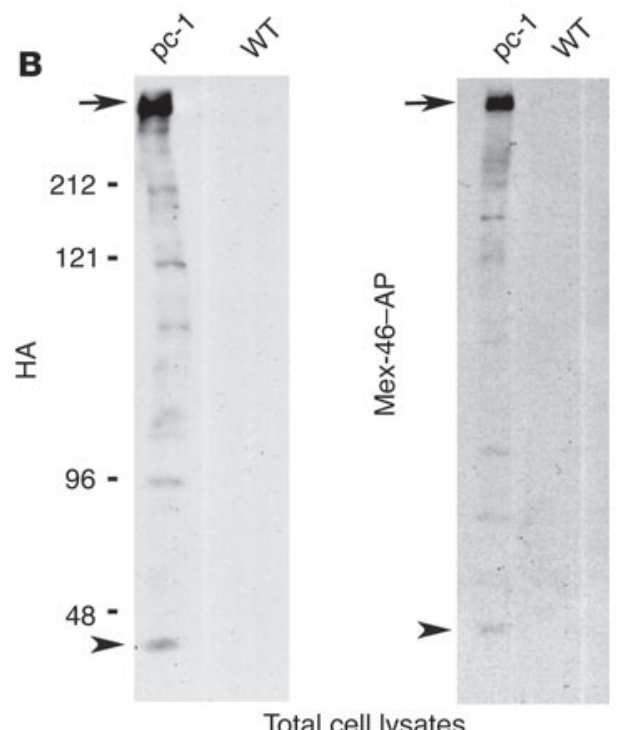

Total cell lysates $\mathrm{HA}$
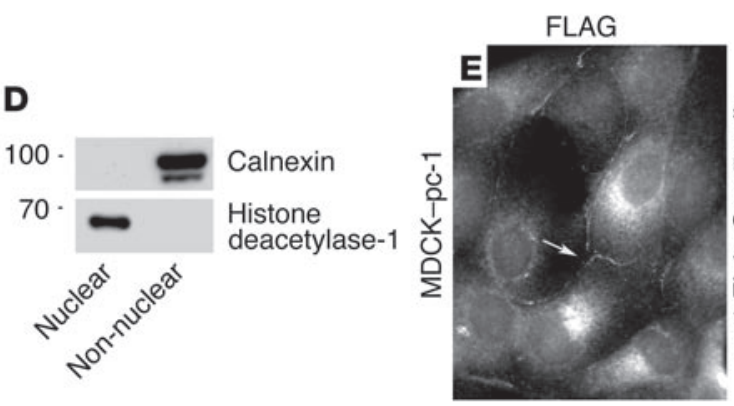

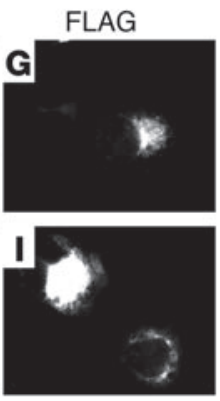

\section{Figure 3}

Expression of full-length polycystin-1 in transfected cells. (A) Schematic of the full-length polycystin-1 construct tagged at its CTT with the HA epitope and at its N terminus (NTF) with a Flag epitope. (B) Western blots of total cell lysates from MDCK cells transfected with the polycystin-1 (pc-1) full-length construct using anti-HA and Mex-46, which specifically recognizes the $\mathrm{C}$ terminus of polycystin- 1 . A highmolecular-weight band is detected by both antibodies in the MDCK cell lines stably transfected with fulllength polycystin-1 (arrows). (C) Nuclear extracts prepared from the same stably transfected MDCK cells as in $\mathbf{B}$ were probed with anti-HA and Mex-46. A band of about $34 \mathrm{kDa}$ is detected in nuclei prepared from transfected cells. Note that a $34-\mathrm{kDa}$ band is also faintly visible in the total lysates (B, arrowheads). No staining is detected in untransfected cells (WT, B and C). (D) The purity of the nuclear fraction examined in panel $\mathbf{C}$, and the completeness of its separation from non-nuclear material, was determined by Western blotting with antibodies directed against the nuclear protein histone deacetylase- 1 and the ER marker calnexin. Left margins (B-D), molecular sizes in $\mathrm{kDa}$. (E) In polycystin-1-transfected MDCK cells, the $\mathrm{N}$-terminal Flag epitope was detected by immunofluorescence microscopy in intracellular membranes and in a punctuate plasma membrane pattern at sites of cell-cell contact. $(\mathbf{F}-\mathbf{I})$ In transfected cell Cos-7 cells, the N-terminal Flag epitope was detected mainly in intracellular membranous structures (G, I). In contrast, the C-terminal HA epitope could also be detected in nuclei $(\mathbf{F}, \mathbf{H})$. Arrows in $\mathbf{E}-\mathbf{I}$ indicate lateral membrane staining. FL, full-length. Original magnification, ×1000 (E-I).

deacetylase-1 (Figure 4C, right panel). A similar pattern was obtained in $\mathrm{CHO}$ cells (not shown). These data demonstrate that a soluble C-terminal fragment of polycystin-1 accumulates in the nucleus.

Further examination of the CTT sequence revealed the presence of a short segment homologous with motifs that have been shown to mediate nuclear localization. This putative nuclear localization sequence (NLS) is composed of two stretches of basic amino acids that lie between residues 4,134 and 4,154 and is highly conserved between species. To test the possibility that the nuclear localization of p200 is attributable to this motif, we generated a construct lacking these 21 amino acids, called p2004 (Figure 4A). When expressed p200). PLAP-VSV-G fusion proteins have previously been shown to target the cytoplasmic domains of a variety of proteins to the cell surface (27) and consequently would be expected to retain the p200 construct outside the nucleus. As expected, the PLAP-p200 construct was not located in the nucleus in transfected cells stained with anti-PLAP or with antipolycystin-1 $\mathrm{C}$ terminus (not shown). In addition, no activation of the AP-1 pathway was observed with this membrane-targeted construct (Figure 4E). These data suggest that at least in the context of the assay used in our study here, activation of the AP-1 pathway by the last 200 amino acids of polycystin-1 requires that it be soluble and have the capacity to enter the nucleus. 
A

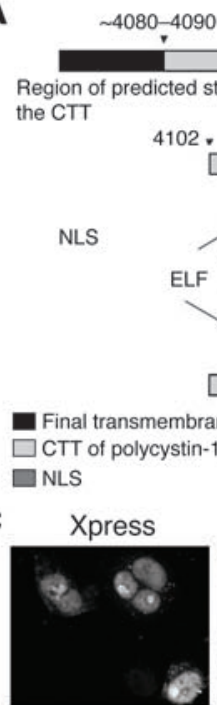

pc-1

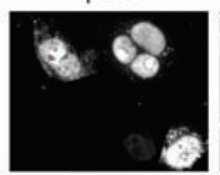

Overlay

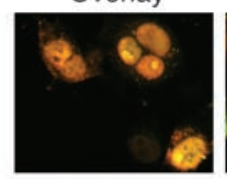

E

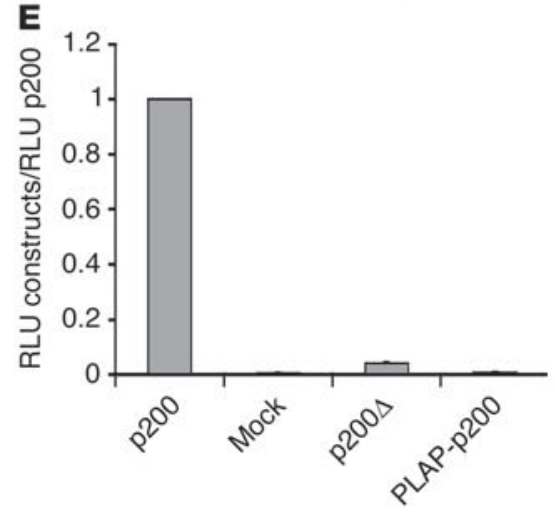

B

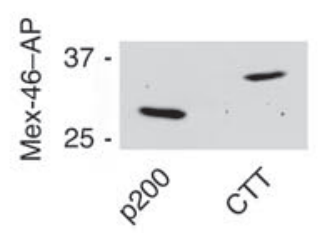

Figure 4

The CTT of polycystin- 1 accumulates in nuclei and activates the AP-1 pathway. (A) Schematic diagram of the structure of polycystin-1 CTT constructs. The predicted CTT includes residues 4,048-4,302. The p200 construct contains the final 200 amino acids of the polycystin- 1 tail, extending from residue 4,102 to residue 4,302 . The putative NLS, located between residues 4,134 and 4,154 , is deleted in the p200 $\Delta$ construct. (B) Comparison of the molecular weights of p200 and the CTT generated from full-length polycystin-1 expressed in MDCK cells. The p200 protein migrates with an apparent molecular weight of about $28 \mathrm{kDa}$, whereas the CTT derived from full-length polycystin-1 migrates as a protein with a molecular weight of about $34 \mathrm{kDa}$. (C) When expressed in Cos-7 cells the p200 protein accumulates mainly in the nucleus, as detected with polycystin-1 and Xpress antibodies (left center panel). Nuclei are labeled with a histone deacetylase-1 antibody (Hd; right center panel). (D) In contrast, the p200 $\Delta$ protein is excluded from the nucleus in transfected Cos-7 cells, as observed with the polycystin-1 and Xpress antibodies (left panels), and distributes instead in a pattern suggestive of association with the ER, as seen by colocalization using Serca-2 and polycystin-1 antibodies (right panels). Original magnification, $\times 1000$ (C and D). (E) Expression of p200 considerably elevates the activity of a coexpressed AP-1 reporter system over the background detected in cells transfected with an empty vector (Mock). This activation is not observed with the p200 $\Delta$ construct or with the PLAP-p200 construct, in which p200 is attached to a membrane anchor. Thus, p200 must be free to enter the nucleus in order to activate the AP-1 pathway. The AP-1 activity for each condition is normalized to the activity observed in p200-expressing cells. RLU, relative light units. Each bar is the average of six experiments performed in triplicate. (F) Although p200 $\Delta$ does not activate AP-1 activity, it is expressed at a level comparable to or greater than that of p200, as determined by Western blotting with an antibody directed against the Xpress tag.

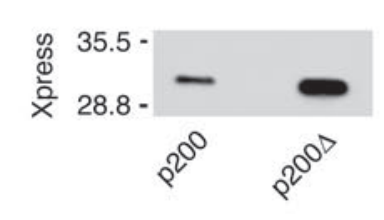

$\mathbf{F}$ directed against the xpress tag.

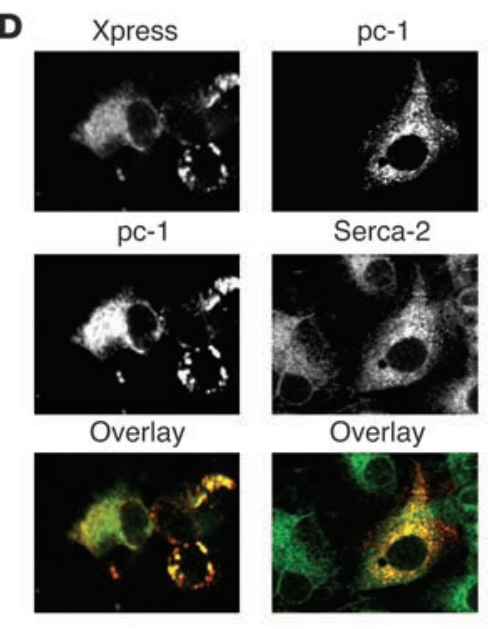

Nuclear access of the cytoplasmic tail of polycystin-1 and activation of the AP-1 pathway are modulated by polycystin-2. To test the possibility that interaction of polycystin- 2 with polycystin- 1 could modulate the nuclear import of the latter's C-terminal domain, we transiently coexpressed full-length polycystin- 2 as well as the p200 construct in Cos-7 cells. Overexpression of polycystin-2 led to the retention of the soluble p200 fragment outside of the nucleus. The p200 construct was distributed in a pattern consistent with localization to the ER in the doubly transfected cells (Figure 5A). We asked whether this retention was associated with a modification in the activation of AP- 1 signaling. Transient expression of the soluble p200 construct and full-length polycystin- 2 along with the AP-1 reporter system led to a reduction in AP-1 activity of 2- to 3-fold compared with the effect of the CTT alone (Figure 5B).
This effect was not due to a reduction in p200 expression, and only a slight diminution in AP-1 activation was observed when the p200 protein was coexpressed with a mutant form of the polycystin-2 protein lacking the domain that interacts with polycystin-1 (Figure 5B) (10). This result suggests that the p200 construct must interact with polycystin-2 for polycystin-2 to exert its inhibitory effects on AP-1 activation. No AP-1 activation was detected after transfection with WT polycystin-2 alone (Figure $5 \mathrm{~B})$. The expression level of the polycystin-2 constructs was also similar in the different experiments (Figure 5B). The relatively small, 2- to 3-fold reduction in AP-1 activity observed when p200 was coexpressed with WT polycystin-2 was probably due to heterogeneity among the transiently transfected cells in the extent to which coexpression of p200 and polycystin-2 was successfully 
A
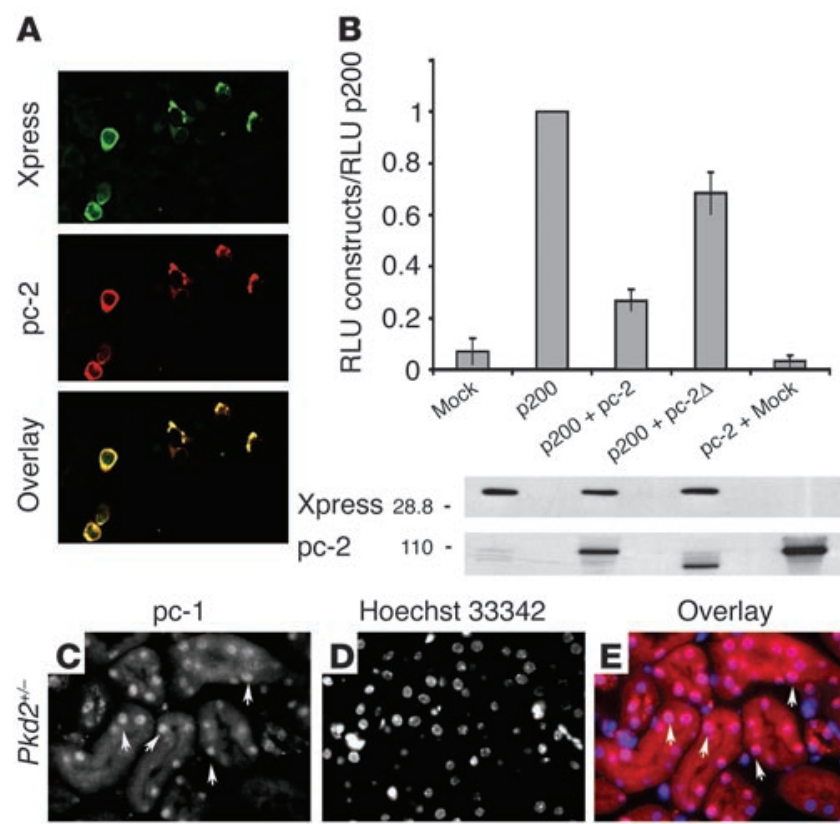

Hoechst 33342
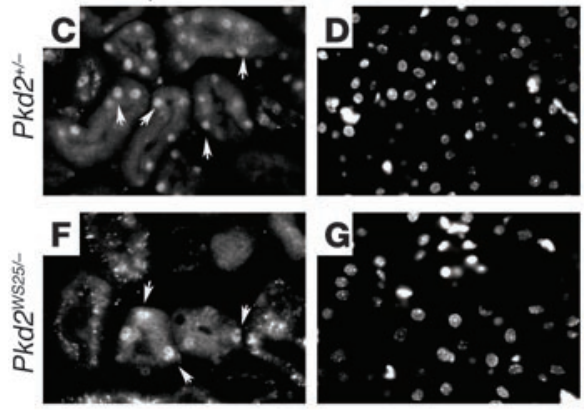

Overlay
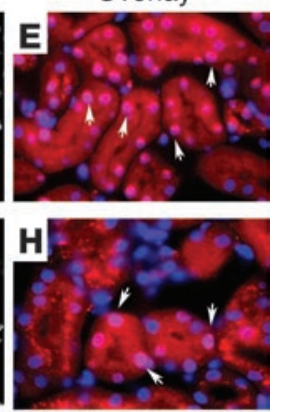

achieved. Immunofluorescence analysis (not shown) revealed that only a subpopulation of the cells were simultaneously transfected with both constructs, whereas some cells were expressed either the p200 or polycystin-2 construct alone.

We next examined whether the level of polycystin-2 expressed in vivo in renal epithelial cells might exert an effect on the nuclear localization of the endogenously expressed polycystin-1 $\mathrm{C}$ terminus. The level of polycystin-2 expression appears to be higher than that of polycystin-1 in adult mouse and human tissues $(17,20)$, and this fact may account in part for the low levels of detection of the polycystin-1 CTT in the nuclei of WT adult kidneys. To address this possibility, we analyzed in vivo the localization of the CTT of polycystin- 1 in mice expressing reduced levels of polycystin-2 (28). Because $P k d 2^{-/-}$mice exhibit an embryonic lethal phenotype, we chose instead to use the $P k d 2^{+/-}$(Figure 5, C-E) and $P k d 2^{W S 25 /-}$ (Figure 5, F-H) mouse models (28). The WS25 Pkd2 gene was created to be an unstable allele that can spontaneously undergo somatic rearrangement (28). Both mice carry one Pkd2-null allele, resulting in reduced expression of polycystin-2. Nuclear localiza-

\section{Figure 6}

Mechanical stimuli induce nuclear localization of the polycystin-1 CTT. (A-F) Kidneys from mice subjected to unilateral ureteral clamping (UUO) were stained with Mex-46 (A and D). Nuclear staining that colocalizes with Hoechst 33342 (B, E, and $\mathbf{H}$ ) is detected in a subset of renal tubular cells in each of the kidneys subjected to clamping, as revealed in the superimposed images (Mex-46: red; Hoechst: blue) (C and F). (D-F) Higher magnification of the regions outlined in the boxes in $\mathbf{A}-\mathbf{C}$. (G-I) No nuclear staining is seen in the contralateral, nonclamped kidney (CL). Original magnifications, $\times 200($ A-C and G-I) and ×750 (D-F).

\section{Figure 5}

Influence of polycystin-2 expression. (A) When coexpressed in Cos-7 cells with full-length polycystin-2 (red), p-200 (green) is excluded from the nucleus and localizes instead to intracellular membranes whose distribution resembles that of the ER. (B) Coexpression of polycystin-2 with p200 decreases the AP-1 activation induced by p200 by a factor of about 2.5 (top). The level of expression of the p200 and pc-2 constructs was determined by Western blotting (bottom). Polycystin-2 $\Delta$ $(p c-2 \Delta)$ refers to a construct that lacks the domain of polycystin-2 that interacts with polycystin-1. (C-H) The CTT of polycystin-1 (stained with Mex-46; C and F) is detected in the nuclei of renal tubular epithelial cells from mice expressing a reduced level of polycystin-2 (C-E, $P k d 2^{+/-} ; \mathbf{F}-\mathbf{H}, P k d 2^{\text {WS25/-); }}$; nuclei were detected with Hoechst 33342 (D and $\mathbf{G})$. (E and $\mathbf{H})$ Superposition of Hoechst (blue) and Mex-46 (red) staining. Original magnifications, $\times 100(\mathbf{A})$ and $\times 750(\mathbf{C}-\mathbf{H})$.

tion of the polycystin-1 cytoplasmic tail was detected in the cells of apparently normal tubules (Figure 5, C-H) in three different mice of each genotype. No nuclear staining was detected with the preimmune serum. Taken together, these data point to a role for polycystin-2 in the regulation of the nuclear localization of the cytoplasmic tail of polycystin-1 in vitro and in vivo, as well as in the modulation of its biological activities.

Mechanical stimuli are associated with the release of the polycystin-1 CTT in vivo. Recent data have highlighted a putative role for the primary cilium in the pathophysiology of different animal models of polycystic kidney disease (PKD) (29-31) and several studies have suggested that it might act as a sensory organelle $(13,32)$. We used two different approaches to determine a possible link between mechanosensation and the potential release of the CTT and its nuclear translocation in vivo. We examined the subcellular distribution of the CTT both after performing unilateral ureteral ligation in WT animals and in a mouse model presenting a defect in the KIF3A subunit of kinesin-II, which has been shown to be essential for cilia formation (33). In the first approach, the clamp was maintained for 2 hours to effectively reduce the tubular flow (34). After this interval, the mice were sacrificed and sections of both kidneys were stained with antibodies against the polycystin-1 $\mathrm{C}$ terminus. The contralateral kidneys, as well as kidneys from anesthetized animals on which no surgery was performed (sham-
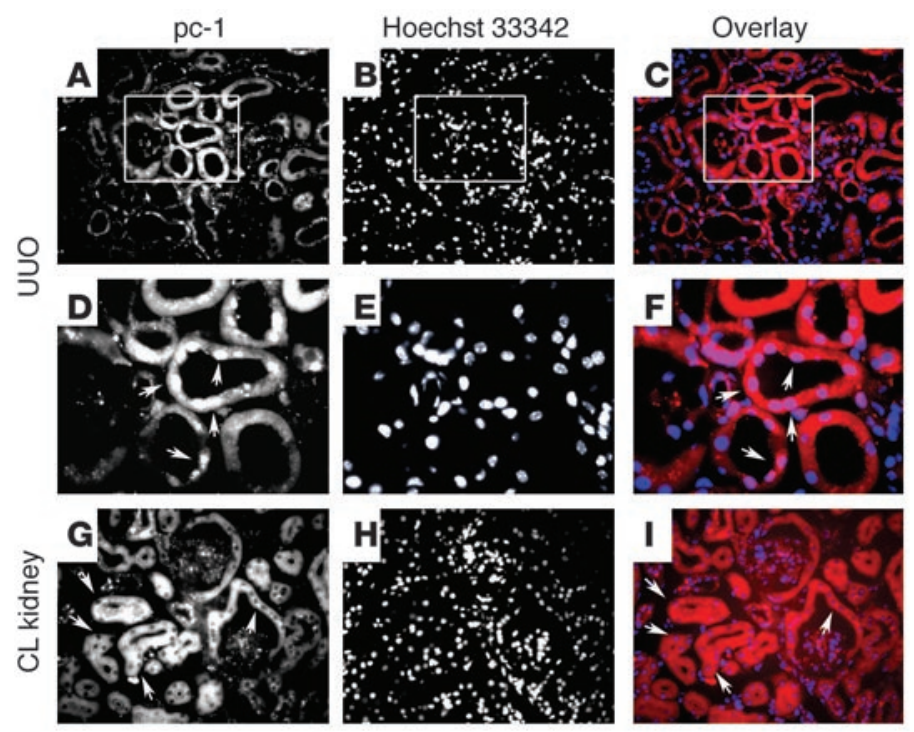


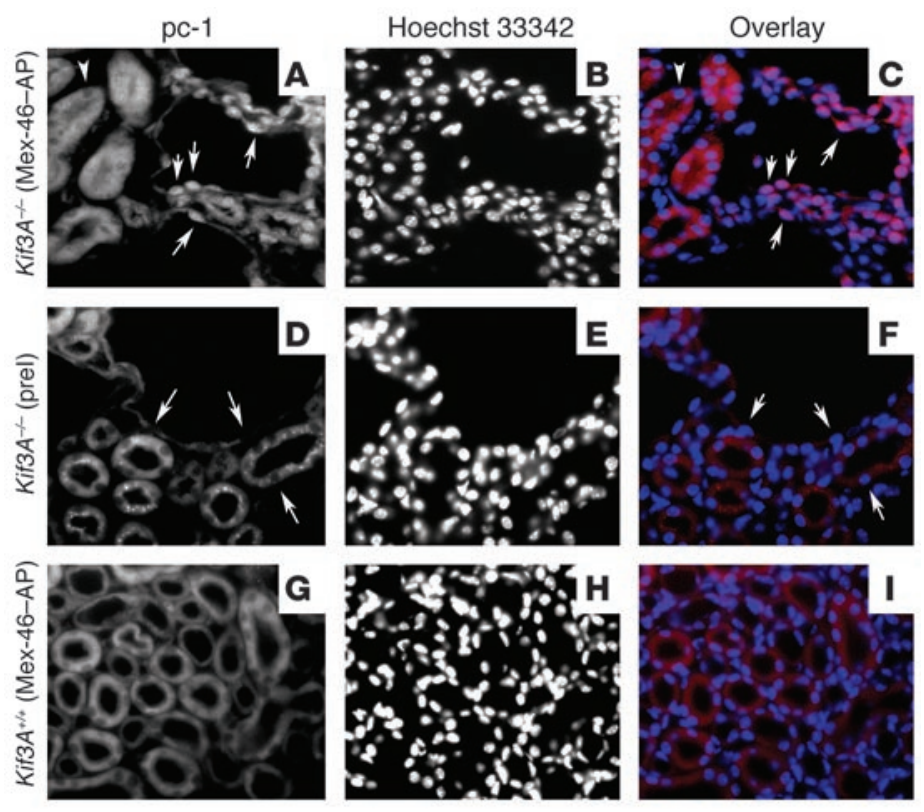

\section{Figure 7}

Mutations that prevent the assembly of cilia are associated with nuclear accumulation of the CTT. Kidneys from KIF3A KO mice $\left(\right.$ Kif3 $\left.^{-/-}\right)(\mathbf{A}-\mathbf{F})$ and WT mice $\left(K i f 3 A^{+/+}\right)(\mathbf{G}-\mathbf{I})$ were examined by immunofluorescence microscopy using Mex-46-AP (A and $\mathbf{G}$ ) or preimmune serum (prel) (D). Nuclei are stained with Hoechst 33342 ( $\mathbf{B}, \mathbf{E}$, and $\mathbf{H}$ ), and superposition of Mex-46-AP (red) and Hochst (blue) staining is depicted in $\mathbf{C}, \mathbf{F}$, and $\mathbf{I}$. In sections prepared from KIF3A KO animals, nuclei (arrows) of cyst epithelial cells, as well as the epithelial cells lining neighboring tubules, are stained with Mex-46-AP (A) but not with preimmune serum (D). No nuclear staining with Mex-46-AP is detected in tubule cells of WT mice (G). treated), were used as controls. After ureteral ligation, nuclear labeling was clearly observed in a subpopulation of nephron segments (Figure 6, A-C and D-F, high magnification), which were identified as distal tubules based upon their colabeling with an antibody against calbindin (not shown). This experiment was repeated in 10 mice and nuclear localization of the polycystin-1 CTT was observed in the ligated kidney of each animal, independently of age (from 3 to 10 months) and sex. No nuclear staining was detected in the contralateral kidneys (Figure 6, G-I) or in the sham-treated animals (not shown).

Kidney-specific inactivation of the Kif $3 A$ gene, using a Cre/loxP strategy in which Cre expression is under the control of the Kspcadherin promoter, results in viable offspring. These animals develop cysts soon after birth, leading to renal failure by postnatal day 21 (33). The cyst epithelial cells lack a primary cilium and exhibit the biological alterations in cell growth associated with PKD. We wondered if the nuclear translocation of the CTT could be detected in the epithelial cells lining the cysts, which do not have a primary cilium. Indeed, the CTT of polycystin-1 was detected in the nuclei of epithelial cells lining the cysts in kidney sections from mice with conditionally inactivated Kif $3 \mathrm{~A}$ (Figure $7, \mathrm{~A}-\mathrm{C})$. No staining was seen with preimmune serum or in WT littermates (Figure 7, D-I).

These data demonstrate that the $C$ terminus of polycystin- 1 is able to access the nucleus in vivo in renal tubular epithelial cells of WT animals. They also suggest that alterations in mechanical stimuli related to renal tubular flow or in the capacity of epithelial cells to sense these alterations can trigger the cleavage events that are involved in this aspect of polycystin-1 signaling.

\section{Discussion}

We have demonstrated that the CTT of polycystin- 1 is present in the nuclei of renal tubular epithelial cells in mice overexpressing the native full-length protein. As polycystin- 1 is an integral membrane protein, this observation suggests that the CTT must be cleaved in order to be translocated to the nucleus. A protein corresponding to the last 200 amino acids of the CTT accumu- lated in the nucleus when it was expressed as a soluble construct, and a functional nuclear localization signal was identified within its sequence. This 21-residue motif (positions 4,134-4,154) is highly conserved among species, varying by a single amino acid between humans and mice (leucine versus phenylalanine, respectively, at position 4,143 ). We have shown that the cleavage of the CTT occurs with expression of full-length polycystin-1 in transfected cells. Although the putative cleavage site is still unknown, the size of the fragment we detected in the nuclei of transfected cells (about $34 \mathrm{kDa}$ ) is consistent with the expected size of the C-terminal cytosolic tail, which contains approximately 200-226 residues (24). The CTT of our polycystin- 1 construct contains 27 additional residues that represent the three HA tags. The p200 construct migrates as a band of $28 \mathrm{kDa}$ in SDS gels and contains a total of 234 residues, including the Xpress tag. Consequently, the cleavage probably occurs close to or within the last transmembrane domain, as it does in proteins that undergo a regulated intramembrane proteolysis. We were unable to detect a specific immunoreactive band in Western blots performed on nuclear fractions prepared from kidneys of WT or transgenic mice. Immunohistochemistry indicated that the nuclear staining was only detectable in a subset of tubule segments. This heterogeneity probably accounts for our failure to detect the polycystin-1 CTT signal contributed by only a subset of renal segments in Western blots of nuclear fractions prepared from whole-kidney homogenates.

The CTT of both human and mouse polycystin- 1 appears to behave in a similar way. All of the CTT constructs were derived from the human sequence, whereas we used the mouse full-length polycystin-1 for establishing the transgenic mouse model and the stable cell lines. Together, these findings suggest that the nuclear translocation of the CTT is likely to be an important event in the polycystin-1 signaling pathway. The release of the $\mathrm{N}$-terminal fragment of polycystin-1 at the $\mathrm{G}$ protein-coupled receptor proteolysis site has been demonstrated recently (14). Further experiments will be necessary to determine whether $\mathrm{N}$-terminal cleavage precedes and is a prerequisite for $\mathrm{C}$-terminal cleavage, as is the case for many 
RIP pathways. Additional work will be also required to identify the nature and the components of the proteolytic complex involved in the release of the polycystin-1 CTT.

The precise identities of the target genes downstream in the polycystin-1 signaling pathway have not been fully characterized. Nevertheless, several studies have suggested that the Wnt (35) and AP-1 pathways are affected by polycystin- 1 signaling $(25,26)$. The transcription factor AP-1 is involved in a variety of processes, including proliferation, transformation, and apoptosis (36). Cell proliferation and apoptosis have been shown to be impaired in cell lines overexpressing full-length polycystin-1 (37) as well as in kidneys from patients with ADPKD (38). Here we have provided further evidence for activation of the AP-1 pathway by polycystin-1 via a novel mechanism. AP-1 reporter gene expression was considerably increased when a soluble construct encompassing the last 200 amino acids of polycystin- 1 and capable of translocating to the nucleus (p200) was overexpressed in cells. This activation was significantly reduced when a construct lacking the putative NLS (p2004) was expressed or when this soluble construct was prevented from entering the nucleus by being expressed as a membrane-bound protein (PLAP-VSV-G-p200). These results underscore the importance of the nuclear localization of the last 200 amino acids of polycystin-1 for the initiation of AP-1 signaling in our system. Previous studies by Arnould et al. (25) reported an activation of the AP-1 pathway when the cytoplasmic tail of polycystin- 1 was targeted to the plasma membrane as a CD16-CD7 fusion protein. The constructs used by those authors included 26 additional amino acids in the membrane-proximal region. The potential for a nuclear localization of the cytoplasmic portion of this polycystin-1 construct was not assessed. Although we did not observe nuclear localization or AP-1 activation when the p200 construct was targeted to the plasma membrane as a PLAP-VSV-G-p200 chimera, it is possible that sequences contained in the constructs used in the study of Arnould et al. are important for the cleavage of the CTT of polycystin-1. Furthermore, it should be noted that in the study of Arnould et al., the assays were performed in a different cell line from those used in our experiments and under different conditions (i.e., serum starvation). The specificity or the activity of the proteolytic complex might vary from one cell line to another.

Parnell et al. (26), using constructs similar to those of Arnould et al. (the last 222 or 226 residues), showed that the activation of the AP-1 pathway by the polycystin-1 cytoplasmic tail was mediated by heterotrimeric $\mathrm{G}$ proteins and PKC. It is possible, therefore, that the constructs used in each of those studies activate AP- 1 through distinct mechanisms, whose relative importance depends upon the precise subcellular localization of the polycystin-1 C-terminus and the particular constellation of environmental stimuli. Consistent with this possibility, we found that although no AP-1 activation was observed with the membrane-bound PLAP-p200 chimera, this construct was able to upregulate expression of p21 (not shown). Similar activation of $\mathrm{p} 21$ expression has been seen with full-length polycystin-1 via activation of the JAK-STAT signaling pathway (39). Thus, the membrane-anchored noncleaved PLAP-p200 protein is biologically active and is able to participate in some, but not all, of the signaling pathways associated with full-length polycystin-1. The precise mechanism through which the last 200 amino acids of polycystin- 1 activate the AP-1 pathway and how it relates to its nuclear translocation remain to be determined. In fact, the JNK reporter system assay that we used is thought to reflect activation events that occur in the cytoplasm rather than in the nucleus. It is possible, therefore, that nuclear translocation of the polycystin-1 CTT alters the expression of genes that participate in modulating AP-1 signaling. It should also be noted that some receptors that can participate in a RIP-like mechanism can also participate in pathways involving classical kinase/phosphatase cascades (40). Thus, RIP-like mechanisms and more traditional signaling modalities are not mutually exclusive and can coexist as alternative means by which a single receptor can send a multitude of messages. More experiments will be needed to determine how the polycystin-1 CTT modulates AP-1 and in what manner its availability for nuclear translocation influences this capacity.

Polycystin- 1 and -2 are believed to participate in a common signaling pathway and have been shown to interact physically with one other. Because they appear to be at least partially distributed in different intracellular compartments $(5,11,23,41)$, their interaction could result from the mobilization of either of the two partners from its initial localization. Our study provides evidence that polycystin-2 impairs the nuclear translocation of the polycystin-1 CTT. Transient coexpression of polycystin- 2 with the CTT prevented nuclear localization of the latter and led to a 2- to 3-fold reduction in AP-1 activation. The polycystin-1 CTT was also detected in the nuclei of renal epithelial cells from two mouse models with reduced polycystin-2 protein levels (i.e., $P k d 2^{+/-}$and $P k d 2^{\text {WS25/- }}$ ). Taken together, these data suggest that polycystin- 2 may act as a buffer regulating the nuclear translocation of the released polycystin-1 C-terminus. A similar mechanism has been well described for the E-cadherin/ $\beta$-catenin/APC tumor suppressor complex. The $\beta$-catenin molecule participates in complexes with E-cadherin, the $\mathrm{T}$ cell factor (TCF) family of transcription factors and the tumor suppressor APC. Its signaling is controlled by competitive interactions among these partners. When bound to E-cadherin at the plasma membrane or to the APC-axin complex in the cytosol, $\beta$-catenin is prevented from interacting with the TCF-type transcription factors (42). According to this model, polycystin-2 may modulate the downstream effects of polycystin-1 on its target genes. Observations using mouse models suggest that the relative balance between polycystin-1 and polycystin- 2 expression might play a critical role in polycystin signaling (ref. 43 and S. Somlo, unpublished observations). The nuclear translocation of the CTT might be dependent upon the relative abundance of both of these proteins as well as upon other potential partners that are as yet unidentified.

A series of recent studies points to a putative role for the primary cilium as a mechanosensor in renal tubular epithelium. Mutations in genes involved in the function or assembly of cilia have been linked to cyst development in mouse models of PKD $(29-31,44)$. Two recent reports have suggested a link between dysfunctional cilia and ADPKD. Polycystin-1 and -2 have been localized to the primary cilium $(11,12)$, and Nauli et al. have shown that the two proteins may function as flow-sensitive mechanosensors (13). Bending the primary cilia of MDCK cells induces an increase in intracellular calcium concentrations (32) through a mechanism that may involve polycystin-2. In light of this potential relationship between polycystin function and flow sensing, it is interesting to note that nuclear translocation of the polycystin-1 CTT was detected in vivo in two relevant mouse models: in renal epithelial cells of WT animals after unilateral ureteral ligation, and in mice manifesting a conditional inactivation of the KIF3A subunit, which is essential for cilia formation. In both of these models, mechanosensory signaling is expected to be altered. In the ureteral 
ligation model, fluid flow is reduced and intratubular pressure is elevated in the affected kidney. In the kinesin $3 \mathrm{~A}$ subunit $\mathrm{KO}$ mouse, any mechanosensation that is dependent upon the cilium should be abrogated. It is important to note that ureteral ligation might possibly affect a number of factors in addition to flow and pressure and may initiate nonspecific injury-related events. Taken together, however, our data suggest that alterations in mechanical stimuli or in their detection correlate with the release of the polycystin-1 CTT in vivo. Under physiological conditions, renal tubular epithelium may be able to sense changes in urinary flow or in shear stress in the lumen through a chain of cellular events that ultimately lead to the release of the CTT. In this setting, the observed nuclear translocation of the CTT of polycystin-1 may initiate cellular responses to such mechanical stimuli.

Both polycystin proteins have been implicated in a wide array of diverse cellular signaling pathways, including cell adhesion (45), control of cell proliferation (37), and mechanotransduction (13). The relative importance of the cleavage and nuclear translocation that we have described, as well as their relationship to or involvement in these other polycystin-associated processes, remain to be determined. It seems likely, however, that this novel pathway is involved in the generation of signals that are critical to the mechanisms through which the polycystin proteins function to maintain the normal architecture of the renal tubules.

\section{Methods}

Constructs. Full-length cDNA encoding mouse Pkd1 was modified to include $\mathrm{N}$-terminal Flag and C-terminal HA epitopes (23) and was subcloned into pcDNA3.1 (Invitrogen). The p200 construct, corresponding to the last 200 amino acids of human polycystin-1 protein, was generated by PCR and was subcloned into pcDNA4/HisMaxA (Invitrogen). The p2004 construct was generated using two sets of PCR primers, with deletion of $63 \mathrm{bp}$ from the p200 construct and removal of the 21 amino acids between residues 4,134 and 4,154. The PLAP-p200 construct was made in pcDNA3.1 (Invitrogen) after subcloning of the last 200 amino acids of polycystin- 1 downstream of the extracellular domain of PLAP and the transmembrane region of the VSV-G. The generation of the human PKD2 constructs has already been described (10). All PCR primer details are available upon request.

Cell culture, transfection, antibodies, and immunofluorescence. Cell lines were grown in $\alpha$-MEM (Invitrogen) (MDCK, CHO and Cos-7) or DMEM (Invitrogen) (HEK) supplemented with $10 \% \mathrm{FBS}$ at $37^{\circ} \mathrm{C}$. Transient transfections were performed using the Lipofectamine 2000 reagent (Invitrogen). Stable cell lines were established using G418 (Invitrogen) for clonal selection. For immunofluorescence experiments, the cells were grown on glass coverslips, fixed in $2 \%$ paraformaldehyde dissolved in PBS, permeabilized in PBS/0.3\% Triton/BSA 0.1\%, blocked in GSDB (16\% goat serum, $120 \mathrm{mM}$ sodium phosphate, $0.3 \%$ Triton X-100, and 450 $\mathrm{mM} \mathrm{NaCl}$ ), and incubated with primary antibody at room temperature. Xpress (Invitrogen) or antibodies specific for the polycystin-1 C terminus (BD3 and 46-2) were used for the detection of human C-terminal constructs. Colocalization studies were performed using antibodies against histone deacetylase-1 (Oncogene Laboratory) and a SERCA-2 Ca pump (Affinity Bioreagents) as nuclear and ER markers, respectively. The fulllength $P k d 1$ construct was detected with monoclonal antibody against HA (1:200 dilution; Covance) and polyclonal antibody against Flag (1:200 dilution; Sigma-Aldrich) as well as with the Mex-46 polyclonal antibody raised against the CTT of mouse polycystin-1 (1:200 dilution). Rhodamine-conjugated anti-rabbit IgG and FITC-conjugated anti-mouse IgG (1:200 dilution; Sigma-Aldrich) were used as secondary antibodies. Coverslips were mounted in Vectashield (Vector laboratories) and were examined using a Zeiss LSM 410 confocal microscope. Contrast and brightness settings were chosen so that all pixels were within the linear range. Images are the product of 8 -fold line averaging.

Polyclonal antibody Mex-46 was raised in rabbits against a fusion protein representing the CTT of mouse polycystin-1. Briefly, cDNA encoding a portion of the C-terminal domain of mouse polycystin-1 (residues 4,139-4,293) was subcloned into pGEX-4T-1 (Amersham Pharmacia) or pMAL-c2 (NEB) to generate a GST-Mex-46 fusion protein or an MBPMex-46 fusion protein, respectively. The GST-Mex-46 fusion protein (about $150 \mu \mathrm{g}$ ) was used for subcutaneous rabbit injections. Affinity purification of Mex- 46 was performed against MBP-Mex- 46 fusion protein to remove contaminating anti-GST, as previously described (15). Polyclonal antibodies against the $\mathrm{C}$ terminus of human polycystin-1 (BD3 and 46-2) (15) and polycystin-2 (YCC2) have been described previously (10).

Immunohistochemistry. WT mice and $P k d 1$ transgenic, $P k d 2^{+/-}$, and $P k d 2^{\mathrm{WS} 25 /-}$ mice were perfused and fixed in periodate-lysine-paraformaldehyde (PLP) (28). Before use, cryostat sections prepared from kidneys from these mice were rehydrated in PBS, then were quenched in $0.5 \mathrm{M} \mathrm{NH}_{4} \mathrm{Cl} / \mathrm{PBS} / 0.1 \% \mathrm{BSA}$, blocked in PBS $/ 0.1 \%$ BSA $/ 10 \%$ goat serum $/ 0.02 \%$ Tween, and incubated for 1 hour at room temperature with primary antibody in the blocking solution (human or murine antibodies against the $\mathrm{C}$ termini, 1:200 dilution; Mex-46-AP, 1:20 dilution). The slides were washed, incubated for 45 minutes with secondary antibody, washed, and mounted as described above. Nuclei were visualized with Hoechst 33342 dye (Molecular Probes) according to the manufacturer's instructions. WT and $P k d 1 \mathrm{KO}$ whole embryos were "snap-frozen." After rehydration with PBS, the cryostat sections were "microwave-treated" according to a standard protocol (20) and were stained as described above. Slides were analyzed with a Zeiss Axiophot microscope equipped with a Zeiss Axiocam HRm CCD camera.

Nuclear preparations and immunoblotting. The nuclear preparation protocol was modified from that of Lin et al. (46). Cells grown in 10-cm dishes were harvested in cold PBS, centrifuged 5 minutes at $500 \mathrm{~g}$, and resuspended in hypotonic buffer ( $10 \mathrm{mM}$ HEPES, $1.5 \mathrm{mM} \mathrm{MgCl}, 10 \mathrm{mM} \mathrm{KCl}$, and a standard cocktail of protease inhibitors). Cells were homogenized in a tight-fitting Dounce homogenizer, chilled on ice for 10 minutes, and then shaken for 15 minutes with an Eppendorf mixer 5432. NP-40 was added ( $1 \%$ final concentration) and the preparations were shaken for an additional 15 minutes. The lysates were centrifuged at $1,500 \mathrm{~g}$ for 5 minutes. The resulting supernatant formed the non-nuclear fractions. The pellets (nuclear fractions) were washed in hypotonic buffer, resuspended in high-salt buffer (20 mM HEPES, $420 \mathrm{mM} \mathrm{NaCl}, 1.5 \mathrm{mM}$ $\mathrm{MgCl}_{2}, 0.2 \mathrm{mM}$ EDTA, 25\% glycerol, and protease inhibitors), shaken for 20 minutes, and finally centrifuged at $14,000 \mathrm{~g}$ for 30 minutes. All steps were performed at $4{ }^{\circ} \mathrm{C}$. Protein concentrations were determined using the Bradford method (Bio-Rad Laboratories). Equal quantities of protein were subjected to PAGE ( $5 \%$ gel for full-length polycystin-1; $12 \%$ for nuclear preparations) and were analyzed with standard Western blot protocols (HRP-conjugated secondary antibodies from Jackson Laboratories and ECL from Amersham). The histone deacetylase-1 antibody obtained from Oncogene Laboratory and the calnexin antibody from Stressgen were used according to the manufacturers' instructions.

Mice. The generation of the Pkd2 ${ }^{\text {Ws } 25 /-}$ and the KIF3A KO mouse models have already been described $(28,33)$. For the generation of $P k d 1$ transgenic mice, bacterial artificial chromosome (BAC) clone 287A3, containing $P k d 1$, was identified by Southern hybridization of the RPCI-22 library arrayed on a grid (ResGen). PCR using the BAC DNA as template confirmed that the clone contained the entire $P k d 1$ gene as well as at least $20 \mathrm{~kb}$ of upstream sequence. BAC DNA was prepared using a standard alkaline lysis protocol, dissolved in Tris plus EDTA buffer, and passed over a QIAGEN Tip-500 endotoxin-free column (Qiagen). BAC DNA 
was eluted using QN buffer (Qiagen), precipitated with isopropanol, and dissolved in $10 \mathrm{mM}$ Tris and $0.1 \mathrm{mM}$ EDTA ( $\mathrm{pH}$ 7.4) for injection. Purified DNA at a concentration of $10 \mathrm{ng} / \mu \mathrm{l}$ was microinjected into the pronuclei of fertilized $\mathrm{C} 57 \mathrm{BL} / 6 \times \mathrm{CD} 1 \mathrm{~F}_{1}$ mouse eggs that were implanted into pseudopregnant females by established protocols of the Transgenic Mouse Service at Yale University (http://info.med.yale.edu/yarc/ transgenic.htm). Five founders containing the BAC DNA were identified by PCR across the junctional region between insert and vector arm using genomic DNA extracted from tail biopsy material. Four of 5 founders passed the transgene through the germline. Northern blot of kidney tissue revealed an increase of about 4-fold in Pkd1 mRNA from 1 founder compared with that of $P k d 1^{+/-}$kidney tissue (data not shown). This mouse line was called $P k d 1^{T g(R P 22-287 A 3) 375 o m}$. To confirm that the BAC transgene in this founder produced functional polycystin-1, we intercrossed $P k d 1^{++-}$: $P k d 1^{T g(R P 22-287 A 3) 375 o m}$ mice to produce $P k d^{-1-}: P k d 1^{\operatorname{Tg}(R P 22-287 A 3) 375 o m}$ offspring. These latter offspring, lacking endogenous polycystin-1 but containing the BAC transgene, were viable with grossly normal organs at weaning, indicating that the BAC transgene expressed functionally active polycystin-1. Animal experiments were performed following review and approval by Yale University's Animal Care and Use Committee.

AP-1 assay. CHO or Cos-7 cells were grown in 60- $\mathrm{mm}$ dishes and were transiently transfected (Lipofectamine 2000; Invitrogen) with a mixture of $0.5 \mu \mathrm{g}$ pFA2-cJun (Stratagene), $0.5 \mu \mathrm{g}$ pFR-Luc (Stratagene), $0.5 \mu \mathrm{g}$ SV $40-\beta-\mathrm{Gal}$, and $6 \mu \mathrm{g}$ of the polycystin-1 C-terminal constructs. Six micrograms of pcDNA 3.1 was used for the mock transfection. For coexpression studies, $3 \mu \mathrm{g}$ of each cDNA (p200 and polycystin- 2 constructs) was used and results were compared with the coexpression of p200 and pcDNA3.1 ( $3 \mu \mathrm{g}$ each). The total amount of DNA used was $7.5 \mu \mathrm{g} /$ dish. After 24 hours, cells were lysed in a reporter lysis buffer (Promega) and luciferase activity was determined with the DualLuciferase reporter assay system (Promega) according to the manufacturer's instructions. Transfection efficiency was normalized by measurement of $\beta$-galactosidase activity. Each assay was performed in triplicate and the results are the average of 6 different experiments.

Unilateral ureteral ligation. Thirteen C57BL/ 6 mice (males and females) were used for this experiment. The ages ranged between 3 and 10 months. Mice were anesthetized by intraperitoneal injection of pentobarbital (100 $\mathrm{mg} / \mathrm{kg}$ body weight). Catheterization of the left jugular vein was performed for intravenous perfusion and the left ureters were ligated at the hilus. After a delay of 2 hours, animals were perfused and fixed with PLP. Sections from both kidneys, clamped and unclamped, were analyzed with antibodies against C-terminal polycystin-1 in association with Hoechst 33342 dye to stain the nuclei as described above. A monoclonal calbindin antibody (Sigma-Aldrich) was used as a marker of distal tubules. Three mice were only anesthetized and perfused without ureteral ligation and were used as controls. The contralateral kidneys in ligated animals were also used as control specimens.

\section{Acknowledgments}

We thank V. Radjendran, S.A. Mentone, S. Nishimura, L. Smith, W.R. Dackowski, and Z. Du for invaluable assistance. This work was supported by a PKD Center grant from the National Institutes of Health (DK 57328; to M.J. Caplan, S. Somlo, and P. Igarashi), and by a Polycystic Kidney Disease Foundation Postdoctoral Fellowship grant (05b2f; to V. Chauvet).

Received for publication March 30, 2004, and accepted in revised form August 17, 2004.

Address correspondence to: Michael J. Caplan, Department of Cellular and Molecular Physiology, Yale University School of Medicine, 333 Cedar Street, New Haven, Connecticut 06510, USA. Phone: (203) 785-7316; Fax: (203) 785-4951; E-mail: michael. caplan@yale.edu.
1. Wilson, P.D. 2004. Polycystic kidney disease. N. Engl. J. Med. 350:151-164.

2. Burn, T.C., et al. 1995. Analysis of the genomic sequence for the autosomal dominant polycystic kidney disease (PKD1) gene predicts the presence of a leucine-rich repeat. The American PKD1 Consortium (APKD1 Consortium). Hum. Mol. Genet. 4:575-582.

3. Mochizuki, T., et al. 1996. PKD2, a gene for polycystic kidney disease that encodes an integral membrane protein. Science. 272:1339-1342.

4. Hanaoka, K., et al. 2000. Co-assembly of polycystin-1 and - 2 produces unique cation-permeable currents. Nature. 408:990-994.

5. Koulen, P., et al. 2002. Polycystin-2 is an intracellular calcium release channel. Nat. Cell Biol. 4:191-197.

6. Tsiokas, L., Kim, E., Arnould, T., Sukhatme, V.P., and Walz, G. 1997. Homo- and heterodimeric interactions between the gene products of PKD1 and PKD2. Proc. Natl. Acad. Sci. U. S. A. 94:6965-6970.

7. Qian, F., et al. 1997. PKD1 interacts with PKD2 through a probable coiled-coil domain. Nat. Genet. 16:179-183.

8. Brown, M.S., Ye, J., Rawson, R.B., and Goldstein, J.L. 2000. Regulated intramembrane proteolysis: a control mechanism conserved from bacteria to humans. Cell. 100:391-398.

9. Urban, S., and Freeman, M. 2002. Intramembrane proteolysis controls diverse signalling pathways throughout evolution. Curr. Opin. Genet. Dev. 12:512-518.

10. Cai, Y., et al. 1999. Identification and characterization of polycystin-2, the PKD2 gene product. J. Biol. Chem. 274:28557-28565.
11. Yoder, B.K., Hou, X., and Guay-Woodford, L.M. 2002. The polycystic kidney disease proteins, polycystin-1, polycystin-2, polaris, and cystin, are co-localized in renal cilia. J. Am. Soc. Nephrol. 13:2508-2516.

12. Pazour, G.J., San Agustin, J.T., Follit, J.A., Rosenbaum, J.L., and Witman, G.B. 2002. Polycystin-2 localizes to kidney cilia and the ciliary level is elevated in orpk mice with polycystic kidney disease. Curr. Biol. 12:R378-R380.

13. Nauli, S.M., et al. 2003. Polycystins 1 and 2 mediate mechanosensation in the primary cilium of kidney cells. Nat. Genet. 33:129-137.

14. Qian, F., et al. 2002. Cleavage of polycystin-1 requires the receptor for egg jelly domain and is disrupted by human autosomal-dominant polycystic kidney disease 1-associated mutations. Proc. Natl. Acad. Sci. U. S. A. 99:16981-16986.

15. Ibraghimov-Beskrovnaya, O., et al. 1997. Polycystin: in vitro synthesis, in vivo tissue expression, and subcellular localization identifies a large membrane-associated protein. Proc. Natl. Acad. Sci. U. S. A. 94:6397-6402

16. Geng, L., et al. 1996. Identification and localization of polycystin, the PKD1 gene product. J. Clin. Invest. 98:2674-2682

17. Geng, L., et al. 1997. Distribution and developmentally regulated expression of murine polycystin. Am. J. Physiol. 272:F451-F459.

18. Guillaume, R., D'Agati, V., Daoust, M., and Trudel, M. 1999. Murine Pkd1 is a developmentally regulated gene from morula to adulthood: role in tissue condensation and patterning. Dev. Dyn. 214:337-348.

19. Guillaume, R., and Trudel, M. 2000. Distinct and common developmental expression patterns of the murine $\mathrm{Pkd} 2$ and $\mathrm{Pkd} 1$ genes. Mech. Dev.
93:179-183.

20. Chauvet, V., et al. 2002. Expression of PKD1 and PKD2 transcripts and proteins in human embryo and during normal kidney development. Am.J. Pathol. 160:973-983.

21. Lieber, T., Kidd, S., Alcamo, E., Corbin, V., and Young, M.W. 1993. Antineurogenic phenotypes induced by truncated Notch proteins indicate a role in signal transduction and may point to a novel function for Notch in nuclei. Genes Dev. 7:1949-1965.

22. Struhl, G., Fitzgerald, K., and Greenwald, I. 1993. Intrinsic activity of the Lin-12 and Notch intracellular domains in vivo. Cell. 74:331-345.

23. Grimm, D.H., et al. 2003. Polycystin-1 distribution is modulated by polycystin-2 expression in mammalian cells. J. Biol. Chem. 278:36786-36793.

24. Nims, N., Vassmer, D., and Maser, R.L. 2003. Transmembrane domain analysis of polycystin-1, the product of the polycystic kidney disease- 1 (PKD1) gene: evidence for 11 membrane-spanning domains. Biochemistry. 42:13035-13048.

25. Arnould, T., et al. 1998. The polycystic kidney disease 1 gene product mediates protein kinase $\mathrm{C}$ alpha-dependent and c-Jun $\mathrm{N}$-terminal kinasedependent activation of the transcription factor AP-1. J. Biol. Chem. 273:6013-6018.

26. Parnell, S.C., et al. 2002. Polycystin-1 activation of c-Jun N-terminal kinase and AP-1 is mediated by heterotrimeric G proteins. J. Biol. Chem. 277:19566-19572.

27. Brown, A.N., Muth, T.R., and Caplan, M.J. 2003. The COOH-terminal tail of the GAT-2 GABA transporter contains a novel motif that plays a role in basolateral targeting. Am. J. Physiol. Cell Physiol. 
286:C1071-C1077

28. Wu, G., et al. 1998. Somatic inactivation of $\mathrm{Pkd} 2$ results in polycystic kidney disease. Cell. 93:177-188.

29. Murcia, N.S., et al. 2000. The Oak Ridge Polycystic Kidney (orpk) disease gene is required for left-right axis determination. Development. 127:2347-2355.

30. Hou, X., et al. 2002. Cystin, a novel cilia-associated protein, is disrupted in the cpk mouse model of polycystic kidney disease. J. Clin. Invest. 109:533-540. doi:10.1172/JCI200214099.

31. Yoder, B.K., et al. 2002. Polaris, a protein disrupted in orpk mutant mice, is required for assembly of renal cilium. Am. J. Physiol. Renal Physiol. 282:F541-F552.

32. Praetorius, H.A., and Spring, K.R. 2001. Bending the MDCK cell primary cilium increases intracellular calcium. J. Membr. Biol. 184:71-79.

33. Lin, F., et al. 2003. Kidney-specific inactivation of the KIF3A subunit of kinesin-II inhibits renal ciliogenesis and produces polycystic kidney disease. Proc. Natl. Acad. Sci. U. S. A. 100:5286-5291.

34. Persson, A.E., Wahlberg, J., Safirstein, R., and Wright, F.S. 1984. The effect of 2 hours of complete unilateral ureteral obstruction on tubulo- glomerular feedback control. Acta Physiol. Scand. 122:35-43.

35. Kim, E., et al. 1999. The polycystic kidney disease 1 gene product modulates Wnt signaling. J. Biol. Chem. 274:4947-4953.

36. Shaulian, E., and Karin, M. 2002. AP-1 as a regulator of cell life and death. Nat. Cell Biol. 4:E131-E136.

37. Boletta, A., et al. 2000. Polycystin-1, the gene product of PKD1, induces resistance to apoptosis and spontaneous tubulogenesis in MDCK cells. $\mathrm{Mol}$. Cell. 6:1267-1273.

38. Lanoix, J., D’Agati, V., Szabolcs, M., and Trudel, M. 1996. Dysregulation of cellular proliferation and apoptosis mediates human autosomal dominant polycystic kidney disease (ADPKD). Oncogene. 13:1153-1160.

39. Bhunia, A.K., et al. 2002. PKD1 induces p21(waf1) and regulation of the cell cycle via direct activation of the JAK-STAT signaling pathway in a process requiring PKD2. Cell. 109:157-168.

40. Ni, C.Y., Murphy, M.P., Golde, T.E., and Carpenter, G. 2001. gamma-Secretase cleavage and nuclear localization of ErbB-4 receptor tyrosine kinase.
Science. 294:2179-2181.

41. Boletta, A., et al. 2001. Biochemical characterization of bona fide polycystin-1 in vitro and in vivo. Am. J. Kidney Dis. 38:1421-1429.

42. Gottardi, C.J., and Gumbiner, B.M. 2001. Adhesion signaling: how beta-catenin interacts with its partners. Curr. Biol. 11:R792-R794.

43. Pritchard, L., et al. 2000. A human PKD1 transgene generates functional polycystin-1 in mice and is associated with a cystic phenotype. Hum. Mol. Genet. 9:2617-2627.

44. Pazour, G.J., et al. 2000. Chlamydomonas IFT88 and its mouse homologue, polycystic kidney disease gene $\operatorname{tg} 737$, are required for assembly of cilia and flagella. J. Cell Biol. 151:709-718.

45. Charron, A.J., Nakamura, S., Bacallao, R., and Wandinger-Ness, A. 2000. Compromised cytoarchitecture and polarized trafficking in autosomal dominant polycystic kidney disease cells. J. Cell Biol. 149:111-124.

46. Lin, S.Y., et al. 2001. Nuclear localization of EGF receptor and its potential new role as a transcription factor. Nat. Cell Biol. 3:802-808. 